\title{
SOP Schädel-Hirn-Trauma (SHT)
}

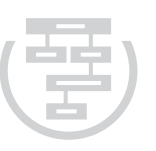

Andreas Pingel, Christoph-Heinrich Hoffmann

\section{Einleitung}

Die Inzidenz der Schädel-Hirn-Verletzungen in Deutschland beträgt ca. 300000/Jahr, wovon etwa 10000 als schwere SHT zu klassifizieren sind. Von diesen versterben trotz guten Traumamanagements und hochentwickelter apparativ-technischer Infrastruktur ca. 2750 Patienten.

Die Behandlung des SHT zielt auf die Minimierung der Sekundärschäden des verletzten Hirns ab, wofür die Weichen schon am Unfallort und im Schockraum gestellt werden. Daher kommt einem standardisierten Management im präklinischen und klinischen Bereich entscheidende Bedeutung zu.

\section{Präoperative Diagnostik und Klassifikation}

\section{Klinische Symptomatik}

Hauptsymptom des SHT ist die Bewusstseinsstörung, diese kann von kurzer, leichter Benommenheit über eine retrograde Amnesie bis hin zum anhaltenden Koma reichen. Das Spektrum der Symptome ist groß und beinhaltet alle möglichen neurologischen Defizite sowie epileptische Anfälle.

Zur Einteilung der SHT nach dem Schweregrad hat sich in den letzten Jahrzehnten die Glasgow Coma Scale - Tab. 1) [26] weltweit durchgesetzt, da hierdurch eine rasche orientierende neurologische Einschätzung des Schädel-Hirn-Verletzten und bei dynamischem Verlauf eine gewisse prognostische Risikoabschätzung möglich sind Das schwere SHT ist mit einem GCS 3-8 definiert, das mittlere SHT mit 9-13 Punkten, von einem leichten SHT spricht man bei einer Punktesumme von 14-15. Bei der Punktzuordnung sind der Einfluss von Intoxikationen (Alkoholintoxikationen oder Drogeneinfluss) oder Medikamenten, aber auch andere konkurrierende Komaursachen zu berücksichtigen.

Im Vordergrund der Therapie steht die Senkung des erhöhten intrakraniellen Druckes, entweder operativ oder durch konservative Maßnahmen.
- Tab. 1 Glasgow Coma Scale (GCS).

\begin{tabular}{|c|c|c|}
\hline Kriterium & beobachtete Reaktion & Punkte \\
\hline \multirow[t]{4}{*}{ Augenöffnen } & spontan & 4 \\
\hline & auf Ansprache & 3 \\
\hline & auf Schmerzreiz & 2 \\
\hline & keine/ohne & 1 \\
\hline \multirow{5}{*}{$\begin{array}{l}\text { beste sprachliche } \\
\text { Antwort }\end{array}$} & voll orientiert & 5 \\
\hline & desorientiert & 4 \\
\hline & inadäquate Äußerung & 3 \\
\hline & unverständliche Laute & 2 \\
\hline & keine & 1 \\
\hline \multirow{6}{*}{$\begin{array}{l}\text { beste motorische } \\
\text { Reaktion }\end{array}$} & adäquat auf Aufforderung & 6 \\
\hline & gezielte Abwehr auf Schmerz & 5 \\
\hline & ungezielte Abwehr auf Schmerz & 4 \\
\hline & Beugesynergismen & 3 \\
\hline & Strecksynergismen & 2 \\
\hline & keine & 1 \\
\hline
\end{tabular}

Merke

Zeichen des erhöhten intrakraniellen Druckes:

- Bewusstseinstrübung

- Schwindel, Übelkeit, Erbrechen, Kopfschmerzen

- neurologisches Defizit, insbesondere Anisokorie

- Cushing-Trias: Bradykardie, Hypertonie, pathologisches Atemmuster

\section{Schockraummanagement}

Die Aufgabe des eingespielten Schockraumteams besteht in der schnellstmöglichen Stabilisierung der Vitalfunktionen und der Erkennung der relevanten Verletzungen sowie der Einleitung einer entsprechenden Behandlung. Die Abläufe müssen standardisiert und darauf ausgerichtet sein, frühzeitig lebensbedrohliche Blutungen oder Hypoxie-Ursachen zu erkennen und eine Akut-cCTDiagnostik oder eine Trauma-Spirale durchzuführen.

Nach Übergabe durch das Rettungsteam erfolgt die Schockraumdiagnostik und -therapie nach dem ATLSKonzept einschließlich einer überblickenden neurologischen Untersuchung (Ansprache, Extremitätenbewegungen, Blick in die Pupillen mit Lichtreaktion, Untersuchung 
des Kopfes bezüglich Wunden, Austritt von Flüssigkeit aus Nase oder Gehörgang).

Blutungen aus Rissquetschwunden des Skalps sollten zunächst durch einen Kompressionsverband provisorisch gestillt werden. Blutungen aus Ohren oder Nase werden tamponiert.

Eine Entfernung von Fremdkörpern aus dem Schädel sollte in jedem Fall unterlassen und nur im Rahmen einer neurochirurgischen Operation vorgenommen werden.

\section{PRAXIS}

Absolute Indikation zum cCT [2]:

- Koma

- Bewusstseinstrübung

- Amnesie

- andere neurologische Störungen

- Erbrechen, wenn ein enger zeitlicher Zusammenhang mit der Gewalteinwirkung besteht

- Krampfanfall

- klinische Zeichen oder röntgenologischer Nachweis einer Schädelfraktur

- Verdacht auf Impressionsfraktur und/oder penetrierende Verletzungen

- Verdacht auf Liquorfistel

- bei Hinweisen auf eine Gerinnungsstörung

Relative Indikation zum cCT [3]:

- unklare Angaben über die Unfallanamnese

- starke Kopfschmerzen

- Intoxikation mit Alkohol oder Drogen

- Hinweise auf ein Hochenergietrauma*

* Fahrzeuggeschwindigkeit $>60 \mathrm{~km} / \mathrm{h}$, erhebliche Deformation des Fahrzeugs, Eindringen von $>30 \mathrm{~cm}$ in die Passagierkabine, Bergungsdauer aus dem Fahrzeug $>20$ min, Sturz > 6 m, Überrolltrauma, Fußgänger- oder Motorradkollision mit > $30 \mathrm{~km} / \mathrm{h}$, Trennung des Fahrers vom Motorrad [3]

\section{PRAXIS}

\section{Schockraummaßnahmen}

- Reevaluation der Vitalparameter und des GCS

- Normotonie, Normoxämie!

- Diagnostik nach ATLS-Richtlinien (Rö-Thorax, Rö-Becken, CTTraumaspirale (CT-Kopf vor KM-Gabe!) oder CCT + CT-HWS

- Neurochirurg im Schockraum!

- Entscheidung über Notoperation oder konservative intensivmedizinische Weiterbehandlung

- Minuten entscheiden! Zeit von Eintreffen bis OP möglichst 20 Minuten

\section{Traumabedingte Ursachen und Pathophysiologie der intrakraniellen Drucksteigerung}

Ursachen einer intrakraniellen Drucksteigerung ( $\bullet$ Abb. 1) sind beim schweren SHT intrazerebrale Blutungen (Kontusionsblutungen) oder extrazerebrale Blutungen (Epiduralhämatome, Subduralhämatome). Daneben kommt es, je nach Grad der Hirnverletzung, begleitend zu einer Hirnschwellung, die zu einer intrakraniellen Drucksteigerung führen kann, wenn die Reserveräume (Blutkompartiment, Liquorkompartiment) ausgeschöpft sind. Die Hirnschwellung führt zu einer Minderperfusion und kann bei Überschreiten eines kritischen Grenzwerts zu einer transienten oder permanenten Ischämie und somit zu Hirninfarkten führen, die ihrerseits wieder den Hirndruck erhöhen ( $\triangleright$ Abb. 1).

Dauer und Ausmaß der Hirndrucksteigerung über $20 \mathrm{mmHg}$ sind unabhängige Prädiktoren eines schlechten Outcomes [15]. Werden die Ursachen der intrakraniellen Drucksteigerung nicht frühzeitig behandelt, können bleibende neurologische Funktionsstörungen unterschiedlicher Schweregrade oder sogar Einklemmungssyndrome des Mittelhirns oder des Hirnstamms mit akuter Lebensbedrohung oder mit schwerwiegenden bleibenden Funktionsstörungen hervorgerufen werden [4].

Beim Mittelhirnsyndrom kommt es durch Scherung des Uncus am mesialen Temporallappen zu einer Kompression des Mesenzephalons und - bedingt durch seine räumliche Nähe - des N. oculomotorius. Deswegen ist die resultierende Pupillendilatation ein Epiphänomen des Mittelhirnsyndroms, welches sich durch Koma und Beugesynergismen auszeichnet.

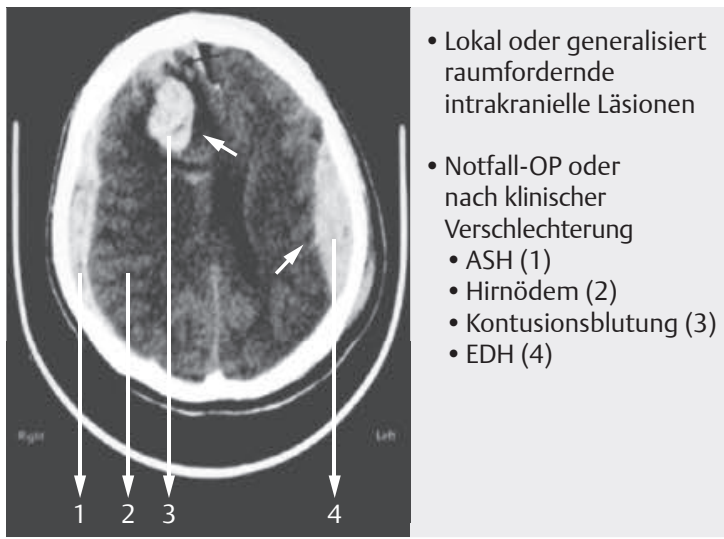

- Abb. 1 Schädel-Hirn-Trauma. Mögliche Ursachen einer intrakraniellen Hirndrucksteigerung: lokal oder generalisiert raumfordernde intrakranielle Läsionen. Notfall-OP oder nach klinischer Verschlechterung: 1: ASH; 2: Hirnödem; 3: Kontusionsblutung; 4: EDH. 
Beim Bulbärhirnsyndrom werden die Kleinhirntonsillen durch das Foramen magnum nach kaudal gegen das Bulbärhirn gepresst, was zu Koma und Strecksynergismen führt.

\section{Operative Therapie des schweren SHT}

\section{PRAXIS}

Allgemeine Indikationen für ein operatives Vorgehen nach SHT

- intrakraniell raumfordernde Blutung, mit Einschränkungen bei tiefen und multifokalen Kontusionsblutungen

- geschlossene Impressionsfrakturen über Kalottenbreite

- offene Impressionsfrakturen, mit Einschränkungen im Sinusbereich

- konservativ nicht zu beherrschender, intrakranieller Druckanstieg > $20 \mathrm{mmHg}$

- frontobasale Frakturen mit Liquorrhö (aufgeschoben dringlich)

- penetrierende SHT (mit Einschränkungen)

\section{Akutes Subduralhämatom}

Das akute Subduralhämatom (ASH) ist die häufigste, akut operativ zu behandelnde Folge eines schweren SHT. Hierbei kommt es zur Zerreißung von Brückenvenen oder kortikalen Arterien, aus denen es in den Subduralraum einblutet, was eine raumfordernde Druckwirkung auf das daruntergelegene Hirn ausübt ( $\mathbf{A} \mathbf{b b} \mathbf{2} \mathbf{2} \mathbf{a})$. Diese Verletzungsform ist häufig assoziiert mit diffusen Hirnkontusionen. Die Hirnkompression führt zu einer oftmals ausgeprägten progredienten Ödembildung. Die Sterblichkeit liegt bei $50-70 \%$.

Es besteht eine Indikation zu einer sofortigen operativen Entlastung bei

- festzustellenden Hirndruckzeichen,

- einer Hämatombreite $>10$ mm,

- einer Mittellinienverlagerung >5 mm, unabhängig vom GCS,

- einem Abfall des GCS um 2 Punkte oder

- einem konservativ nicht zu beeinflussenden ICP $>20 \mathrm{mmHg}$.

Hierbei sollten unmittelbar nach Indikationsstellung eine großflächige Trepanation frontotemporoparietal mit kompletter Hämatomausräumung sowie ggf. eine Duraerweiterungsplastik erfolgen, um dem nachschwellenden Hirngewebe den entsprechenden Schwellraum zu gewähren ( $\bullet$ Abb. $\mathbf{2}$ b). Ein Wiedereinbringen des Kraniotomiedeckels sollte in aller Regel nicht im Rahmen der Akutintervention erfolgen. Der Kalottendeckel kann zur
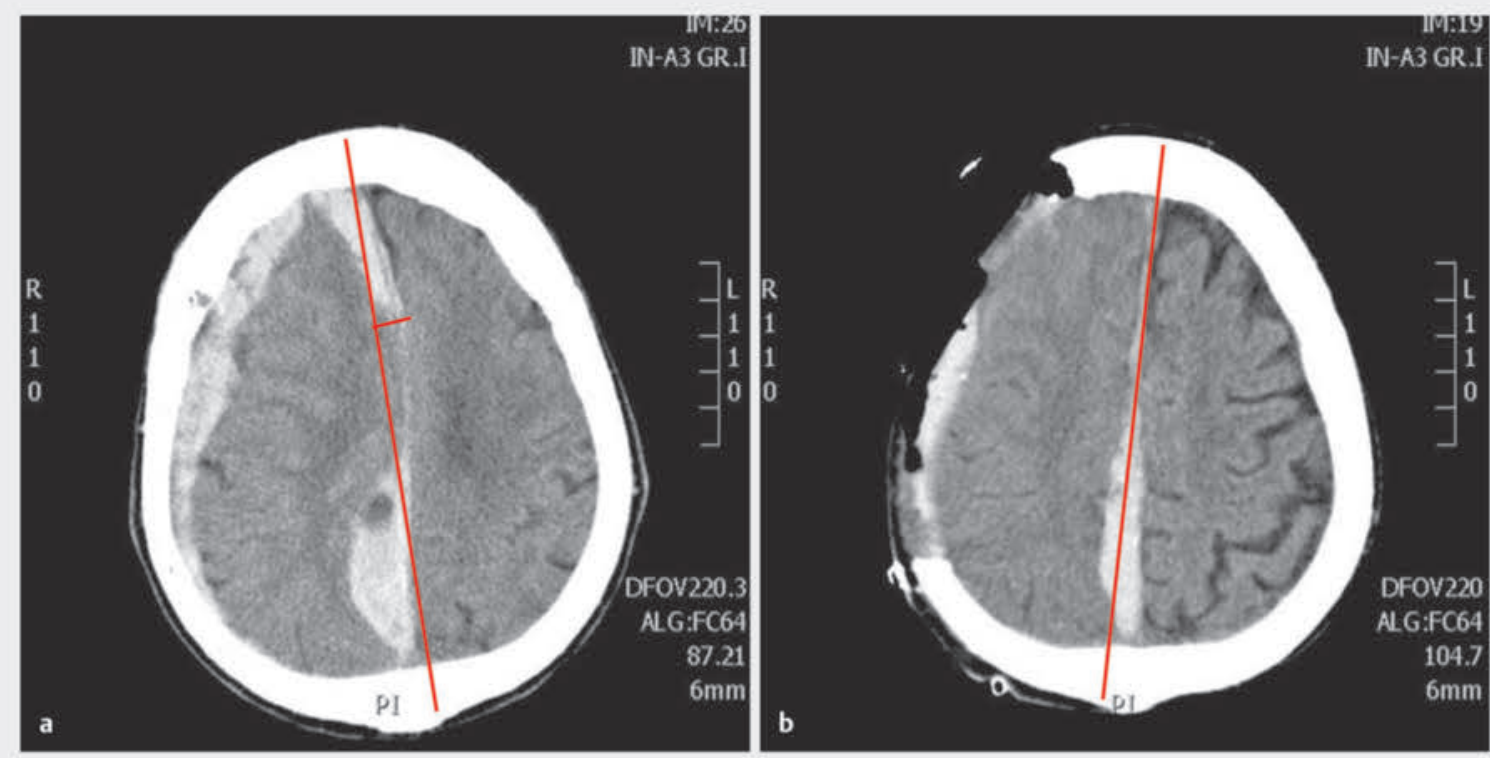

- Abb. 2 Schädel-Hirn-Trauma. Akutes Subduralhämatom rechts.

a Vor operativer Dekompression.

b Nach operativer Dekompression. 

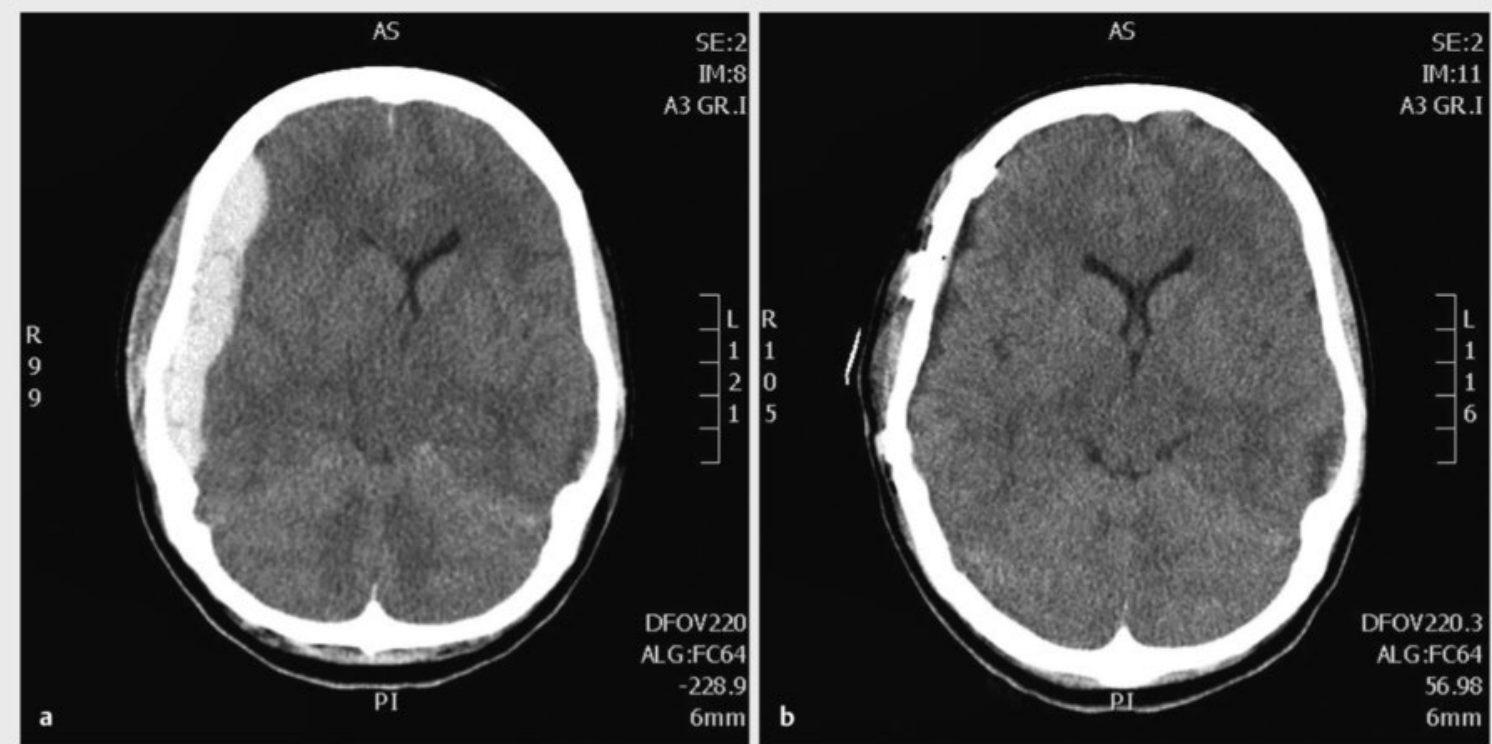

- Abb. 3 Schädel-Hirn-Trauma. Epiduralhämatom (EDH) rechts frontal.

a Vor der Dekompression.

b Nach der Dekompression.

späteren Reimplantation kryokonserviert oder in eine subkutane abdominale Tasche implantiert werden. Die Schädeldachplastik erfolgt in der Regel nach 3-6 Wochen, wenn das Hirngewebe klinisch und computertomografisch sichtbar abgeschwollen ist und das Kalottenniveau nicht mehr überragt.

\section{Epiduralhämatom}

Epiduralhämatome (EDH) sind in der Regel die Folge lokalisierter Krafteinwirkungen auf den Kopf und zu 90\% mit einer Schädelfraktur assoziiert. Ursachen sind eine ZerreiBung von Ästen der Meningealarterien oder Blutungen aus Frakturspalten. Das EDH weist im cCT eine typische bikonvexe Form auf, die Lokalisation ist zu ca. 75\% temporal. EDH betreffen eher jüngere Verletzte, jenseits des 65. Lebensjahrs ist es durch die großflächige Verklebung der Dura mit der Kortikalis interna der Schädelkalotte selten zu finden.

Bei einem Volumen von über $30 \mathrm{ml}$ und einer Hämatombreite von über $15 \mathrm{~mm}$ sowie einer Mittellinienverlagerung von mehr als $5 \mathrm{~mm}$, unabhängig vom GCS, sollten Epiduralhämatome notfallmäßig operativ entlastet werden. Diese CT-Kriterien besitzen nach Bullock [5] einen prädiktiven Wert für das Outcome.

Bei der operativen Versorgung ist auf eine akribische Blutstillung zu achten, insbesondere, wenn die A. meningea media Blutungsquelle ist. Die Kraniotomie sollte idealerweise der Ausdehnung des Epiduralhämatoms entsprechen. Der Kalottendeckel kann am Ende der OP meist wieder replantiert werden ( $\bullet$ Abb. $\mathbf{3}$ ).

Bei wachen Patienten, welche die oben genannten Kriterien nicht erfüllen, kann eine konservative Behandlung mit klinischer Überwachung und cCT-Verlaufskontrolle nach 6-8 Stunden erfolgen.

\section{Intrazerebrale Kontusionen}

Intrazerebrale Kontusionen sind Folgen eines schweren SHT und häufig zusammen mit traumatischen Subarachnoidalblutungen und akuten Subduralhämatomen anzutreffen. In etwa $60 \%$ aller schweren SHT sind Kontusionen nachzuweisen. In Parenchymzonen mit gestörter BlutHirn-Schranke kann es bei gleichzeitiger Zerreißung kleiner parenchymaler Gefäße zur Formierung von Hämatomen, bevorzugt frontal und temporal, in der Nähe der direkten Krafteinwirkung sowie auf der Gegenseite (contre coup) kommen. Diese können nach Stunden oder Tagen zu größeren Hämatomen konfluieren, was durch die Zunahme der raumfordernden Wirkung häufig mit einer klinischen Verschlechterung einhergeht. Daher ist neben einer engmaschigen neurologischen Beobachtung ein Kontroll-cCT nach 6-12 Stunden oder unmittelbar bei Verschlechterung durchzuführen.

Bei großen raumfordernden Blutungen mit drohender Einklemmung ist eine Blutungsausräumung, ggf. auch im Sinne einer dekompressiven Hemikraniektomie, häufig lebensrettend. Eine Indikation zur Blutungsausräu- 
mung wird im Allgemeinen ab einem Blutungsvolumen von $20 \mathrm{ml}$ und einer Mittellinienverlagerung (MLV) von mehr als $5 \mathrm{~mm}$ gesehen.

In ca. 15\% der schweren SHT sind intraventrikuläre Blutungen zu finden, die zu einem Hydrozephalus führen können. Bei CT-gesichertem Liquoraufstau ist eine externe Ventrikeldrainage (EVD) anzulegen.

\section{Dekompressive (Hemi-)Kraniektomie}

Wenn nach Ausschöpfung aller konservativen Maßnahmen zur Hirndrucksenkung weiterhin pathologisch erhöhte Hirndrücke (ICP > $20 \mathrm{mmHg}$ ) kontinuierlich bestehen, ist die Durchführung einer dekompressiven (Hemi-) Kraniektomie ein- oder beidseitig bzw. subokzipital eine effektive Maßnahme [20]. Hierdurch wird dem nachschwellenden Hirn Raum zum Ausweichen gegeben, und die zerebrale Mikrozirkulation kann verbessert werden. Die drohende und lebenslimitierende transtentorielle bzw. Foramen-magnum-Herniation kann hierdurch häufig abgewendet werden ( $\bullet$ Abb. 4).

Während besonders bei Kindern und Jugendlichen die Indikation zur dekompressiven Kraniektomie großzügig gestellt wird, sollte sie bei Patienten über 55 Jahren und bei Hinweisen auf ausgedehnte irreversible Hirnschäden mit Zurückhaltung erfolgen $[12,14]$.

Der Durchmesser der Kraniektomie sollte mindestens 10-12 cm betragen, um einen Volumengewinn von etwa $30 \mathrm{~cm}^{3} \mathrm{zu}$ erreichen. Bei einer maximalen Kraniektomie ist ein Volumengewinn von $100 \mathrm{~cm}^{3}$ möglich [25]. Bei der dekompressiven (Hemi-)Kraniektomie ist die Durchführung einer über die gesamte Trepanationsfläche ausgedehnten Duraeröffnung essenziell.

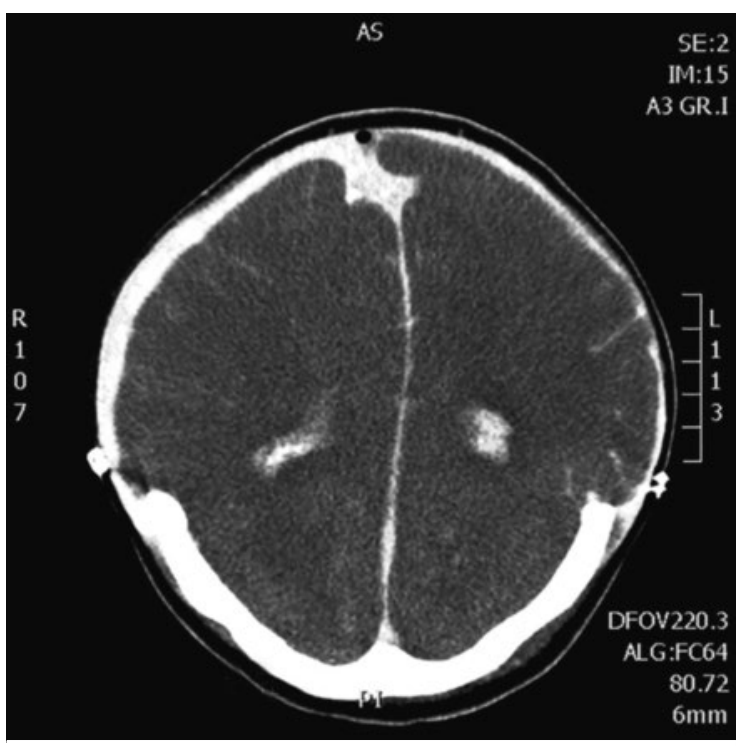

- Abb. 4 Schädel-Hirn-Trauma. Bilaterale Kraniektomie.

\section{Schädelbasisfrakturen}

\section{Rhino-/Otoliquorrhö}

Während eine geringe Liquorrhö in aller Regel spontan sistiert, muss eine anhaltende oder kräftige Liquorrhö oder eine Verletzung mit Austritt von Hirnparenchym operativ plastisch gedeckt und so in eine geschlossene Verletzung umgewandelt werden. Eine prophylaktische Antibiotikagabe wird im Allgemeinen nicht empfohlen.

Bei Felsenbeinfrakturen sollte eine HNO-Evaluation erfolgen, ggf. kann durch Rheologika eine Hypakusis oder eine Commotio labyrinthi positiv beeinflusst werden.

Bei knöcherner Beteiligung der Orbita oder des Optikuskanals, bei Auffinden eines Retrobulbärhämatoms oder bei Symptomen wie Doppelbildern oder Visusminderung bei wachen Patienten sollten augenärztliche Untersuchungen vorgenommen werden.

\section{Traumatische Subarachnoidalblutung}

Die traumatische Subarachnoidalblutung (SAB) ist bei schwerem SHT häufig. Sie stellt an sich keine Operationsindikation dar, geht aber oft mit einem traumatischen Hirnödem einher und kann zu einem Vasospasmus der hirnversorgenden Arterien und damit zu Ischämien führen. Eine SAB ohne Traumaanamnese muss angiografisch weiter abgeklärt werden, um eine Gefäßmalformation als Blutungsursache auszuschließen.

\section{Konservative und postoperative Therapie des schweren SHT}

Ziele der konservativen und postoperativen Behandlung sind die Vermeidung einer zerebralen Perfusionsstörung und die effektive Senkung eines erhöhten intrakraniellen Druckes (ICP).

Die zerebrale Perfusion muss durch einen zerebralen Perfusionsdruck (CPP = MAP - ICP) von $60-70 \mathrm{mmHg}$ aufrecht erhalten werden [10]. Trotz ausreichenden zerebralen Perfusionsdruckes kann es durch pulmonale Einschränkungen zu einer zerebralen Hypoxie kommen, weshalb ein PEEP bis $10 \mathrm{mmHg}$ erlaubt ist und zusätzlich die periphere Sauerstoffsättigung zwingend beobachtet werden muss. Zudem kann der Sauerstoffpartialdruck im Hirnparenchym durch spezielle Messsonden fortlaufend erfasst werden.

Störungen der zerebralen Autoregulation und Vasospasmen infolge traumatischer Subarachnoidalblutungen können mithilfe regelmäßiger transkranieller Doppler-Sonografien detektiert werden. Das Management des CPP und der Hydratation (Normovolämie) ist den dabei gewonnenen Befunden anzupassen [9]. 


\section{PRAXIS}

\section{Maßnahmen zur Senkung des intrakraniellen Druckes}

- Liquorableitung über EVD

- Analgosedierung (Midazolam, Propofol, Fentanyl, Sufentanil)

$[1,14,21]$

- 30-Oberkörperhochlagerung, Sicherstellung eines ungehinderten venösen Abstroms über die Jugularvenen

- Mannitol 20\% (Serum-Osmolalität < 320 mosmol/l), Furosemid, THAM (Tris-Hydroxymethyl-Aminomethan) [22,23]

- Barbiturate [19]

- Normothermie, ggf. moderate Hypothermie $\left(34-35^{\circ} \mathrm{C}\right)$, aber: erhöhtes Pneumonierisiko! [2, 6, 11]

- kurze, leichte Hyperventilation ( $\mathrm{paCO}_{2} 30-35$ mmHg, < 1 h); Cave: zerebrale Ischämie durch Vasokonstriktion!

Bei bewusstlosen bzw. analgosedierten Patienten ist die fortlaufende Messung des intrakraniellen Druckes obligat $[8,17]$. Sie ist Voraussetzung für eine zeitnahe therapeutische Intervention. Der ICP kann über intraparenchymatöse Sonden reliabel erfasst werden. Bei hinreichend weiten Ventrikeln ermöglicht eine intraventrikulär platzierte externe Drainage (EVD) gleichzeitig die Senkung des ICP.

Die parenterale Ernährung muss dem nach einem SHT um 20-50\% erhöhten zerebralen Energieumsatz [18] frühzeitig angepasst werden. Normoglykämie kann die Prognose hirnverletzter Patienten signifikant verbessern [27].

Der Einsatz von Antikonvulsiva ist auf die symptomatische Therapie von früh auftretenden epileptischen Anfällen beschränkt. Eine frühe prophylaktische Gabe hat keinen Einfluss auf das Auftreten von Spätepilepsien [5, 24].

\section{Prognose}

Trotz aller intensivmedizinischen Maßnahmen ist die Prognose des schweren SHT weiterhin schlecht. Bei optimaler medizinischer Versorgung liegt heute die Letalität des schweren SHT (GCS <9) bei 24\% [18]. 10-30\% sind schwer und 17-20\% mittelgradig behindert. Nur ca. 727\% der Patienten erholen sich gut.

\section{Aufklärung und Komplikationen}

In der Notfallsituation entfällt die Notwendigkeit der Aufklärung. Angehörige sollten dennoch über die Verletzung und die Erfordernis von Operationen informiert werden.

Wache, aufklärungsfähige Patienten werden über das Verletzungsmuster, die Indikation zur Operation und die inhärenten Risiken und Komplikationsgefahren aufgeklärt:

\section{PRAXIS}

\section{Checkliste zur Patientenaufklärung}

- Verbleiben oder Verschlechterung einer neurologischen Symptomatik (Paresen, Sensibilitätsstörungen, Seh-/Hör-/Geruchs-/Schmeckstörungen, Bewusstseinsstörungen, Vigilanzminderung bis zum Koma, Konzentrations-/Gedächtnisstörungen, Sprachstörungen, Wesensänderung, neuropsychologische Symptome)

- Nachblutung

- Entzündungen (intrakranieller Abszess, epi-/ subdurales Empyem), Wundheilungsstörung

- Entwicklung eines Hirnödems mit Notwendigkeit intensivmedizinischer konservativer oder operativer Maßnahmen

- Krampfanfälle

- Liquorfistel mit Notwendigkeit zur Revision

- Entwicklung eines Hydrozephalus mit Notwendigkeit einer temporären oder dauerhaften Liquorableitung (Shunt)

- bei dekompressiver Kraniektomie: spätere Schädeldachplastik

- Lagerungsschäden

- postoperative Thrombose, Embolie

\section{Präoperative Checkliste}

Vor dem Eingriff müssen vorhanden sein:

- aktuelle cCT-Bildgebung zur Seitenverifikation sowie Größen- und Lagebestimmung der anzugehenden Pathologie, darauf basierend Festlegung des operativen Zugangswegs [7, 13]

- Labor, ggf. Ausgleich einer Gerinnungsstörung beginnen!

- Erythrozytenkonzentrate nach erwarteter Blutungsmenge kreuzen (i.d. R. mindestens zwei Konzentrate)

- Reevaluation des Pupillenstatus unmittelbar präoperativ

\section{Perioperative Maßnahmen}

- Lagerung des Kopfes auf der Kopfschale oder in der Mayfield-Klemme, bei einseitiger Kraniotomie Lage der ipsilateralen Schulter erhöht mit untergeschobenem Tuch, leichte Anti-Trendelenburg-Lagerung, Vermeiden einer Kompression der Jugularvenen

- Haarrasur entsprechend der Größe des geplanten Eingriffs

- Kraniotomiesieb, High-Speed-Drill, Sauger, Hirnwatten, Knochenwachs, Hämostyptika (z. B. Gelitta, Tabotamp), Kopfschwartenclips (z. B. Scalpfix), Duraersatzplastik-Materialien (z. B. Tissudura, Neuropatch), monopolarer und bipolarer Kauter, ggf. Miniplates oder Craniofix zur Refixierung des Knochendeckels 
- Kryokonservierung $\left(-70^{\circ} \mathrm{C}\right)$ oder Implantation des entnommenen Knochendeckels in abdominaler Subkutantasche bei dekompressiver Kraniektomie

- intraoperativ ggf. Mannitolgabe, ggf. Anlage einer intraparenchymatösen Hirndrucksonde oder einer externen Ventrikeldrainage zur Messung und Kontrolle des ICP

- unmittelbar postoperativ: Kontrolle des Pupillenstatus

\section{Postoperative Maßnahmen und Nachsorge}

Postoperative Anordnungen:

- Darf Analgosedierung beendet werden?

- weitere hirndrucksenkende Maßnahmen (Oberkörperhochlagerung, Mannitol, Auswahl geeigneter Sedativa, Analgetika)

- keine Antikoagulation

- Zeitpunkt des postoperativen Kontroll-cCTs festlegen

Bei (zu erwartendem) Hirnödem ist die fortgesetzte Analgosedierung mit spezifischer hirndrucksenkender Therapie obligat. Ein Kontroll-cCT wird dann regelmäßig spätestens nach 24 Stunden durchgeführt, um Nachblutungen oder eine Größenzunahme von Kontusionen auszuschließen. Nach Einnahme von Gerinnungsinhibitoren (speziell Kumarinen) sind regelmäßige Kontrollen der Gerinnungsparameter notwendig, um einem erneuten $A b$ fall frühzeitig entgegenzuwirken.

\section{Merke}

Im postoperativen Verlauf ist auf einen ausreichenden CPP zwischen 60 und $70 \mathrm{mmHg}$ zu achten. Ein entsprechendes Blutdruckmanagement ist essenziell.

Bei großflächigen Kraniektomiedefekten muss eine Lagerung auf der Defektzone unbedingt vermieden werden. Selbstredend sind Kompressionsverbände kontraindiziert.

Der Zeitpunkt des postoperativen Weanings ist vom Ausmaß der Hirnverletzung abhängig zu gestalten. KontrollcCTs sind hier nach Maßgabe der klinischen Entwicklung zu terminieren, wobei mindestens eine postoperative cCT-Untersuchung zur Dokumentation des Behandlungsergebnisses notwendig ist.

\section{Merke}

Die Unterbringung der Patienten mit SHT sollte generell auf der Intensivstation erfolgen, da nur hier ein hinreichendes und der individuellen Situation der Patienten angepasstes Monitoring der klinischen Befunde möglich ist.
Interessenkonflikt

Die Autorinnen/Autoren geben an, dass kein Interessenkonflikt besteht.

Autorinnen/Autoren

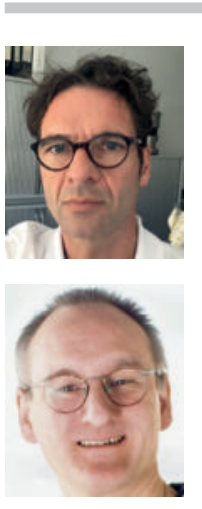

\section{Andreas Pingel}

Dr. med.; Zentrum für Wirbelsäulenchirurgie und Neurotraumatologie, Berufsgenossenschaftliche Unfallklinik Frankfurt am Main.

\section{Christoph-Heinrich Hoffmann}

Dr. med.; Zentrum für Wirbelsäulenchirurgie und Neurotraumatologie, Berufsgenossenschaftliche Unfallklinik Frankfurt am Main.

Korrespondenzadresse

Dr. med. Andreas Pingel

BG Unfallklinik Frankfurt am Main gGmbH

Friedberger Landstraße 430

60389 Frankfurt am Main

andreas.pingel@bgu-frankfurt.de

\section{Zitierweise für diesen Artikel}

Orthopädie und Unfallchirurgie up2date 2021; 16: 11-18. Pingel A, Hoffmann CH. Schädel-Hirn-Trauma (SHT). In: Günther KP, Hoffmann R, Hrsg. SOPs in der Orthopädie und Unfallchirurgie. Stuttgart: Thieme; 2017: 73-80.

Literatur

[1] Albanese J, Viviand X, Potie F et al. Sufentanil, fentanyl and alfentanil in head trauma patients: a study on cerebral hemodynamics. Crit Care Med 1999; 27: 407-411

[2] Alderson P, Gadkary C, Signorini DF. Therapeutic hypothermia for head injury. Cochrane Database Syst Rev 2004; (4): CD001048

[3] AWMF, Arbeitsgemeinschaft der Wissenschaftlichen Medizinischen Fachgesellschaften. ATLS, Advanced Trauma Life Support

[4] Baethmann A, Kempski O, Unterberg A. Entstehung und Therapie zerebraler Sekundärschäden. Münch Med Wschr 1982; 43: 941-944

[5] Bullock R, Chesnut RM, Clifton G et al. Guidelines for the management of severe head injury. Brain Trauma Foundation. Eur J Emerg Med 1996; 3: 109-127

[6] Clifton GL, Miller ER, Choi SC et al. Lack of effect of induction of hypothermia after acute brain injury. N Engl J Med 2001; 344: $556-563$

[7] Connolly ES, McKhann II GM, Huang J et al. Fundamentals of operative Techniques in Neurosurgery. New York: Thieme Medical Publishers; 2002: 563-583 
[8] Fakhry SM, Trask AL, Waller MA et al. IRTC Neurotrauma Task Force: Management of brain-injured patients by an evidencebased medicine protocol improves outcomes and decreases hospital charges. J Trauma 2004; 56: 492-493

[9] Fernandez R, Firsching R, Lobato R et al. Guidelines for treatment of head injury in adults. Opinions of a group of neurosurgeons. Zentralbl Neurochir 1997; 58: 72-74

[10] Brain Trauma Foundation. Guidelines for the management of severe traumatic brain injury“. Update-Erklärung, März 2003

[11] Haselsberger K, Pucher R, Auer LM. Prognosis after acute subdural or epidural hemorrhage. Acta Neurochir (Wien) 1988; 90: $111-116$

[12] Holtkamp M, Buchheim K, Unterberg A et al. Hemicraniectomy in elderly patients with space occupying media infarction: improved survival but poor functional outcome. J Neurol Neurosurg Psychiatry 2001; 70: 226-228

[13] Jallo J, Loftus CM. Neurotrauma and critical Care of the Brain. New York: Thieme Medical Publishers; 2009: 175-237

[14] Maas Al, Dearden M, Teasdale GM et al. EBIC-guidelines for management of severe head injury in adults. European Brain Injury Consortium. Acta Neurochir 1997; 139: 286-294

[15] Marmarou A, Anderson RI, Ward JD. Impact of ICP instability and hypotension on outcome in patients with severe head trauma. J Neurosurg 1991; 75: 59-66

[16] Muizellar JP, Marmarou A, Ward JD et al. Adverse effects of prolonged hyperventilation in patients with severe head injury: a randomized clinical trial. J Neurosurg 1991; 75: 731739

[17] Palmer S, Bader MK, Qureshi A et al. The impact on outcomes in a community hospital setting of using the AANS traumatic brain injury guidelines. Americans Associations for Neurologic Surgeons. J Trauma 2001; 50: 657-664

[18] Piek J. Ernährungstherapie. In: Piek J, Unterberg A. Grundlagen neurochirurgischer Intensivmedizin. München: Zuckschwerdt; 1999: 259-268

[19] Roberts I. Aminosteroids for acute traumatic brain injury (Cochrane Review). In: The Cochrane Library, Issue 1. Chichester, UK: John Wiley \& Sons; 2004
[20] Sahuquillo J, Arikan F. Decompressive craniectomy for the treatment of refractory high intracranial pressure in traumatic brain injury. Cochrane Database Syst Rev 2006; (1): CD003983

[21] Sanchez-Izquierdo-Riera JA, Caballero-Cubedo RE, Perez-Vela JL et al. Propofol versus midazolam: safety and efficacy for sedating the severe trauma patient. Anesth Analg 1998; 86: 1219-1224

[22] Schwartz ML, Tator CH, Rowed DW et al. The University of Toronto head injury treatment study: a prospective, randomized comparison of pentobarbital and mannitol. Can J Neurol Sci 1984; 11: 434-340

[23] Smith HP, Kelly DL jr., McWhorter JM et al. Comparison of mannitol regimens in patients with severe head injury undergoing intracranial monitoring. J Neurosurg 1986; 65: 820-824

[24] Schwartz ML, Tator CH, Rowed DW et al. The University of Toronto head injury treatment study: a prospective, randomized comparison of pentobarbital and mannitol. Can J Neurol Sci 1984; 11: 434-340

[25] Steiger HJ, Reulen HJ. Manual Neurochirurgie. Landsberg: Ecomed; 1999: 299-302

[26] Teasdale G, Jennett B. Assessment of coma and impaired consciousness. Lancet 1974; 2: 81-83

[27] Van den Berghe G, Wouters P, Weekers F et al. Intensive insulin therapy in the critical ill patient. N Engl J Med 2001; 345: 1359-1367

\section{Bibliografie}

Orthopädie und Unfallchirurgie 2021; 16: 11-18

DOI 10.1055/a-1129-7855

ISSN 1611-7859

(c) 2021. Thieme. All rights reserved.

Georg Thieme Verlag KG, Rüdigerstraße 14,

70469 Stuttgart, Germany 